\title{
émulations
}

\section{Approches géographiques de la nuit urbaine libre et festive}

\section{Les free parties et les zones autonomes temporaires (TAZ) comme alternative à la ségrégation et aux inégalités territoriales nocturnes}

Alexandre Grondeau et Gwenaëlle Dourthe

Émulations - Revue de sciences sociales

2020, n³3, «La nuit urbaine. Un espace-temps complexe entre opportunités et inégalités ».

\section{Article disponible à l'adresse suivante}

https://ojs.uclouvain.be/index.php/emulations/article/view/grondeau

\section{Pour citer cet article}

Alexandre Grondeau et Gwenaëlle Dourthe, « Approches géographiques de la nuit urbaine libre et festive. Les free parties et les zones autonomes temporaires (TAZ) comme alternative à la ségrégation et aux inégalités territoriales nocturnes) 》, Émulations, $n^{\circ} 33$, Mise en ligne le 15 juin 2020.

DOI : 10.14428/emulations.033.05

Distribution électronique : Université catholique de Louvain (Belgique) : ojs.uclouvain.be

(c) Cet article est mis à disposition selon les termes de la Licence Creative Commons Attribution, Pas d'Utilisation Commerciale 4.0 International. http://creativecommons.org/licenses/by-nc/4.0/

Éditeur : Émulations - Revue de sciences sociales / Presses universitaires de Louvain https://ojs.uclouvain.be/index.php/emulations

ISSN électronique : 1784-5734

$\frac{\text { PUL PRESSES }}{\text { UNIVERSITAIRES }}$ 


\title{
Approches géographiques de la nuit urbaine libre et festive
}

\author{
Les free parties et les zones autonomes temporaires \\ (TAZ) comme alternative à la ségrégation \\ et aux inégalités territoriales nocturnes
}

Alexandre Grondeau' ${ }^{1}$ et Gwenaëlle Dourthe ${ }^{2}$

\begin{abstract}
[Résumé] Notre article étudie le mouvement free party et ses usagers comme des porteurs de réponses à la standardisation, à la marchandisation et à l'uniformisation du monde de la nuit, mais également comme producteurs d'un nouveau type de droit à la ville et de zones autonomes temporaires (temporary autonomous zones, TAZ). La free party, ses usagers et les territoires qu'ils génèrent seront ainsi appréhendés comme un moyen de lutter contre les discriminations socioéconomiques, culturelles ou vestimentaires inhérentes à la mercantilisation des lieux nocturnes festifs. Pour ce faire, nous analyserons les pratiques et les mécanismes d'appropriation de l'espace des participants aux free parties afin de poser les bases d'une approche géographique de la nuit urbaine, libre et festive.
\end{abstract}

Mot-clés : free party, nuit urbaine, TAZ, DIY.

Geographical approaches of the free and festive urban night: Free parties and temporary autonomous zones (TAZ) as an alternative to segregation and territorial inequalities at night

[Abstract] Our article examines free party movements and their users as an answer to the standardization of the urban night world, but also as producing a new type of right to the city and temporary autonomous zones (TAZ). The free party, its users and the territories they generate are understood as a means of combating socio-economic, cultural or clothing discriminations inherent to the commercialization of festive nocturnal places. We analyze the appropriation mechanisms of these festive spaces to lay the foundations for a geographic approach to the urban night, free and festive.

Key words: free party, urban night, TAZ, DIY.

\section{Introduction}

À partir du début des années 1980, alors que la ville industrielle devenait progressivement la ville néolibérale et ludique (Burgel, Grondeau, 2015), on a pu assister à une remise en question du rôle socioculturel de la nuit (dormir, se reposer, être au calme, profiter de sa vie privée, etc.). Les pratiques, les usages, les représentations de la nuit ont ainsi évolué avec l'apparition de nouvelles centralités et territorialités (Gwiazdzinski, 2014 ; 2015), de nouvelles sociabilités, de nouvelles manières de consommer. Sous l'effet de la conversion des politiques publiques à des logiques managériales (Harvey, 1989 ;

\footnotetext{
${ }^{1}$ Laboratoire TELEMMe UMR 7303, Aix-Marseille Université, France.

${ }^{2}$ Laboratoire TELEMMe UMR 7303, Aix-Marseille Université, France.
} 
2014 ; Peck, 2014) et de la baisse des budgets publics (Brenner, Theodore, 2002), le néolibéralisme et le mercantilisme se sont ainsi totalement emparés de la nuit à travers sa mise en scène (Straw, 2001), sa mise en fête (Gravari-Barbas, 2000), son évènementialisation (Gwiazdzinski, 2007), le développement des partenariats public/privé (Renard, 2008), et sa marchandisation (Crozat, Fournier, 2005).

C'est dans ce contexte de développement du capitalisme nocturne, puis du néolibéralisme du monde de la nuit, que se sont formées de manière ponctuelle des zones autonomes temporaires (temporary autonomous zones, TAZ) (Bey, 1991), incarnations territoriales et éphémères du refus de la ségrégation et des inégalités nocturnes. L'accès payant aux boîtes de nuit et aux salles de concert, le filtrage de leur entrée par des physionomistes ou des services de sécurité, et le formatage progressif de la musique passée dans ces lieux ont généré chez de nombreux jeunes une envie de faire la fête ailleurs et autrement. Se déroulant dans des interstices urbains, dans des squats, des terrains vagues, les free parties vont ainsi se développer un peu partout en Europe et, avec elles, une nouvelle forme de territoires alternatifs proposant des normes et des valeurs festives différentes de celles pratiquées dans la société de consommation nocturne. Dans cet article, nous entendons la free party comme un rassemblement festif illégal, se déroulant sur un ou plusieurs jours et nuits, libre en termes d'accès, et où le genre musical écouté principalement est électronique. Il se différencie de la rave party par son caractère non marchand (entrée gratuite ou sur donation), sa dimension expérimentale et son absence d'encadrement institutionnel.

Notre article étudiera ainsi le mouvement free party et ses usagers comme porteurs de réponses à la standardisation et à l'uniformisation du monde de la nuit (Collette, 2002 ; Gicquel, 2007; Pourtau, 2005), mais également comme producteurs d'un nouveau type de droit à la ville et de zones autonomes temporaires. La free party, ses usagers et les territoires qu'ils génèrent seront ainsi appréhendés comme une proposition alternative aux discriminations socioéconomiques, raciales ou vestimentaires inhérentes à la mercantilisation des lieux nocturnes festifs.

Pour mener à bien notre étude, après un cadrage théorique revenant sur les notions de nuit urbaine festive et de néolibéralisme urbain, nous nous baserons sur les résultats d'une enquête réalisée par questionnaire en ligne en 2018 auprès de 1663 usagers de zones autonomes temporaires ${ }^{3}$, et sur deux enquêtes qualitatives basées sur des entretiens semi-directifs réalisés en France, en 2018 et 2019, auprès de quarante-six membres historiques et actuels du mouvement free party . Notre objectif sera de pré-

\footnotetext{
${ }^{3}$ Nous avons pris le parti d'utiliser cette méthodologie malgré les biais qu'elle peut engendrer, notamment au niveau des simplifications pouvant résulter du traitement des réponses aux questions fermées (que nous avons limitées en pratiquant un grand nombre d'entretiens semi-directifs) et découlant du caractère en ligne du questionnaire (certains anciens activistes étant beaucoup moins connectés que la nouvelle génération). Dans ce dernier cas également, les entretiens semi-directifs ont permis d'intégrer leurs points de vue et leurs vécus.

${ }^{4}$ Sur les quarante-six interviewés, dix-sept étaient encore présents dans le milieu free party (le reste s'étant éloigné de l'activisme musical), quinze résidaient dans un grand quart sud-est, neuf dans un grand quart sud-ouest, neuf résidaient à Paris et en région parisienne, sept dans un grand quart nord-ouest, et six dans un grand quart nord-est.
} 
senter, dans un premier temps et d'un point de vue théorique, l'avènement d'un néolibéralisme urbain nocturne festif et ses différentes déclinaisons territoriales. Dans un second temps, nous chercherons à identifier les pratiques socioculturelles et territoriales alternatives proposées par le mouvement free party. Nous étudierons enfin les mécanismes d'appropriation spatiale que l'on observe dans ce dernier pour le considérer comme une déclinaison du droit à la ville, un droit à la ville libre et festive, mais plus encore comme une nouvelle de forme de TAZ.

\section{Approches géographiques de la nuit urbaine festive, du néolibéralisme territorial et de leurs impacts socioterritoriaux}

La géographie de la nuit festive est un objet d'étude récent, à la croisée de plusieurs approches disciplinaires, et dont les contours ont largement évolué ces trente dernières années, en particulier sous l'effet combiné des processus de mondialisation (Carroué, 2002 ; Grataloup, 2015), de métropolisation (Lacour, Puissant, 2000 ; Burgel, Grondeau, 2015) et de mise en spectacle de nos sociétés et de nos territoires (Debord, 1992 [1967). La convergence de ces trois processus a abouti à une transformation de la manière dont la société, les sciences humaines et la géographie ont appréhendé la relation entre ville, nuit et fête.

$\mathrm{Au}$ tournant des années 1980, la ville industrielle est progressivement devenue ludique, elle s'est mise en scène, a cherché à être attractive et compétitive d'un point de vue économique et, dans ce cadre-là, elle a développé son côté by night, encouragée par le développement et la généralisation des activités liées aux loisirs, la fête en particulier, et à leur évènementialisation (Crozat, Fournier, 2005). Les travaux portant sur la géographie de la fête ont ainsi montré de quelle manière les fêtes et les festivals étaient apparus comme de nouveaux facteurs de production ou de requalification des espaces urbains permettant la mise en place de vastes opérations de labellisation ou de marketing territorial (Bonnemaison, 1990 ; Di Meo, 2005 ; Gravari-Barbas, 2009). La ville est ainsi devenue divertissement (Burgel, 1993), une fun city (Gravari-Barbas, 2000), une happy city (Ballas, 2013) dont la vocation est de distraire autant que de créer de l'activité économique et du rayonnement médiatique.

À partir du début des années 1990, la marchandisation progressive des nuits urbaines festives a engendré l'apparition de nouveaux territoires et territorialités, de nouvelles centralités définissant de nouvelles limites et donc de nouvelles frontières (Gwiazdzinski, 2014). Deux approches théoriques complémentaires permettent alors de mieux cerner les évolutions de la géographie de la nuit festive urbaine : le tourisme urbain nocturne (Giordano, Nofre Mateu, Crozat, 2018) et la night-time economy (Chatterton, Hollands, 2003).

L'étude du tourisme urbain nocturne englobe l'ensemble des pratiques touristiques exercées la nuit. Elles concernent essentiellement les jeunes sans pour autant s'y limiter. Les analyses sur le tourisme urbain trouvent leur origine dans l'étude des stations 
touristiques festives (Bell, 2008 ; Camprubi, Prats, 2013 ; Capellà i Miternique, 2017 ; Fjaer, Tutenges, 2017), puis elles se sont intéressées à la privatisation et à l'évènementialisation des pratiques festives. L'étude du tourisme urbain nocturne permet alors de bien appréhender les mécanismes collectifs d'appropriation territoriale éphémère de publics caractéristiques (les jeunes fêtards), leur caractère initiatique par exemple, tout en s'intéressant à leur potentielle intégration à des politiques d'attractivité territoriale.

C'est justement ce cadre-là que la notion de night-time economy a permis de développer en s'intéressant à l'ensemble des pratiques des collectivités visant à améliorer l'attractivité d'un quartier et d'une ville ou à revaloriser, régénérer, requalifier un territoire urbain en difficulté grâce à des activités de loisirs festifs. Introduite au début des années 1990 au sujet des centres-villes britanniques marqués par la désindustrialisation et régénérés grâce à des activités festives nocturnes, l'expérience s'est généralisée partout dans le monde (Chatterton, Hollands, 2003) en s'accompagnant de certaines caractéristiques : initiation ou renforcement de processus de gentrification, augmentation des prix festifs, encadrement des festivités (horaires définis, sécurisation des lieux), privatisation de certains lieux festifs à l'origine accessible à tous, mise en scène spectaculaire et monumentalisation des lieux nocturnes festifs, caractère générique progressif de ces quartiers festifs au fur et à mesure de leur aménagement dans les métropoles du monde entier et remises en cause et contestations sociales des éléments précédemment cités (Oloukoï, Guinard, 2016).

L'ensemble de ces approches semble indiquer que la marchandisation progressive des nuits urbaines festives correspond peu ou prou à l'application des grands principes politiques du néolibéralisme territorial aux villes et aux quartiers festifs : développement des partenariats public-privé, privatisation des espaces publics, formatage des lieux, criminalisation et/ou stigmatisation de la pauvreté, ultra-sécurisation et fragmentation des espaces, évènementialisation des politiques, etc. (Harvey, 1989, 2014 ; Peck, 2014 ; Ward, 2003). La géographie de la nuit urbaine festive a clairement été modelée ces dernières décennies par ces politiques urbaines néolibérales.

C'est ainsi que l'on retrouve dans ces territoires festifs nocturnes la production de nombreuses interdictions (heure de fermeture), de ségrégation (sélection de certains types de personnes (manière de s'habiller, coiffure, couleur de peau), de censure de certains types de musique expérimentale (hardcore, tribe, jungle, etc.), de privatisation des lieux et d'inégalités territoriales nocturnes (prix des places). Les teuffeurs ${ }^{5}$ ont alors trouvé une réponse territoriale à ces problèmes dans l'organisation de free parties.

\section{Free party : les pratiques d'une fête libre et populaire à l'opposé des fêtes marchandes et filtrées}

« Je ressentais le monde des boîtes de nuit comme l'exact opposé de la manière selon laquelle j'avais envie de faire la fête. C'est aussi simple que cela. Je ne voulais pas faire la

\footnotetext{
${ }^{5}$ Un teuffeur est un participant à une teuf, fête en verlan. Le terme est utilisé pour qualifier les participants à une free party.
} 
queue avec des gens formatés pour payer pour entrer dans des endroits formatés écouter de la musique affreuse. J'avais envie d'autres choses, de liberté », résume P., membre d'un sound system actif à la fin des années $1990^{6}$. Le mouvement free party est apparu à la fin des années 1980 en Grande-Bretagne. Sa composition est protéiforme tant il regroupe d'activistes musicaux issus de différentes franges de la contre-culture britannique : traveller, squatters, ravers, héritiers de la beat generation, post-punk, rude boy, membres de scène underground électronique (Bouyxou, Delannoy, 1995 ; Collette, 2002 ; Maffesoli, 1993 ; Petiau, 2006 ; Tessier, 2003).

L'intense répression gouvernementale concernant l'encadrement du monde de la nuit festive (notamment la fermeture des boîtes de nuit à $2 \mathrm{~h}$ du matin) aboutira à l'inculpation pour « conspiracy to cause public nuisance » de membres du sound system Spiral Tribe suite au festival libre de Castlemorton ${ }^{7}$ (1992) et à l'établissement du Criminal Justice and Public Act en 1994. Ce dernier élargit notamment très largement le pouvoir de la police britannique quant aux arrestations des teuffeurs, aux gardes à vue et aux saisies du matériel sonore. En réponse à cette répression, une partie de ces activistes musicaux prendra la route pour diffuser dans toute l'Europe sa musique, son style de vie et son slogan : «Free Music for Free People».

Organisé en communautés artistiques anarchistes nomades (appelées tribes) voyageant en camion et organisant régulièrement, grâce à leurs systèmes de sonorisation mobiles appelés sound systems, des fêtes libres, éphémères, avant-gardistes et illégales, le mouvement free party va s'affirmer comme une alternative possible aux pratiques festives nocturnes classiques (Epstein, 2001 ; Petiau, 2011 ; Pourtau, 2005) : « La free était un exutoire, un moyen de me shooter à la liberté que tu n’avais pas dans la société de consommation. En y allant, on disait "vous nous faites chier tous les jours en disant ce qu'il faut faire, comment il faut travailler, se comporter, ce qu'il faut manger, ben là pendant deux jours et deux nuits on va pas dormir et on vous emmerde" », explique Y. usager des fêtes libres entre 1995 et 2005.

Dès son origine, la free party se positionne comme un événement en rupture avec les normes et les valeurs du système capitaliste dominant qu'elle appréhende comme un danger contre lequel il faut lutter (Baudrillard, 1997 ; Kosmicki, 2008 ; Petiau, 2006 ; Queudrus, 2001 ; Tessier, 2003), une fête clandestine à l'opposé des pratiques marchandes classiques. B. ${ }^{9}$, activiste électronique depuis la fin des années 1980, précise : " Je venais de la zone, je me suis toujours construit contre le rapport à l'argent. Depuis mes débuts dans ce milieu, le but était de créer un contre-pouvoir et de montrer qu’il

\footnotetext{
${ }^{6}$ Les prénoms de tous les interviewés ont été anonymisés. Entretien avec P., Nice, 15 octobre 2018. ${ }^{7}$ Le festival de Castelmorton est un festival de musique britannique gratuit qui s'est déroulé entre le 22 et le 29 mai 1992 et à l'issue duquel une partie des membres du sound system Spiral Tribe a été arrêtée pour différentes charges, dont trouble à l'ordre public. Ils sont acquittés plus tard, mais la législation britannique contre le monde de la nuit et de la fête se durcit très fortement après cette histoire.

${ }^{8}$ Entretien avec Y., Nice, 18 octobre 2018.

${ }^{9}$ Entretien téléphonique avec B., 5 décembre 2018.
} 
n'y avait pas de fatalité mercantile dans ce monde. " T. ${ }^{10}$, ancien membre d'une tribe historique, explique : «Il y avait forcément un fondement politique à notre démarche : sortir du carcan, d'un système capitaliste dominant et oppressant qu'on avait tous subi et qu'on subissait au quotidien. Quand une free était finie, on ne rentrait pas dans nos appartements bouffer McDo. On était dans nos camions, sur la route, ou dans des squats... » D. ${ }^{11}$, participant régulier des frees entre 1998 et 2006, confirme : « Les tribes étaient composées de gens à part du système qui voulaient créer un monde parallèle sans règles autres que les leurs. » M. ${ }^{12}$ va dans le même sens : " Il y avait un côté mystique en free, une sorte de détachement du monde matériel, une élévation spirituelle, le refus des valeurs qu'on nous avait inculquées depuis l'enfance. »

La plupart des premiers activistes du mouvement free party véhiculent et développent des valeurs clairement en opposition à la société de consommation. Ils organisent leurs événements dans des lieux marginaux (squats, casernes abandonnées, friches industrielles) dont ils ne sont pas propriétaires et qu'ils occupent de manière illégale. La free party apparaît ainsi comme contraire aux principes de propriété privée et favorable à l'usage collectif et non marchand des espaces dont elle a besoin. Les réquisitions festives de terrains sur lesquels va être organisée une free party se réalisent en fonction des opportunités du moment : base militaire abandonnée à Tarnos (1995), plateau de Larzac (2000), piscine abandonnée Molitor (Paris, 2001), zone montagneuse (col de l'Arche, 2002), ou tout simplement un champ en friche, une usine désaffectée, ou une plage facile d'accès (comme à Port-la-Nouvelle en 1998). À chaque fois, la free party va générer un certain nombre de pratiques socioculturelles alternatives (troc, do it yourself-DIY) auxquelles ses usagers peuvent adhérer totalement, partiellement ou pas du tout, selon leurs souhaits. L'espace d'un instant, les teuffeurs s'estiment libres d'agir comme bon leur semble : «On était dans notre bulle. Chacun faisait ce qu'il voulait, tant qu'il n'emmerdait pas les autres », explique M. ${ }^{13}$.

Parmi ces pratiques multiples, alternatives à la néolibéralisation du monde de la nuit urbaine festive, les plus emblématiques sont le troc, l'autogestion, le do it yourself et, en premier lieu, la création et la promotion de la liberté consubstantielle à l'événement lui-même. Contrairement à une idée reçue et véhiculée dans certains médias, la free party n'est pas exclusivement synonyme de fête gratuite. Elle se positionne avant tout comme une fête libre. Le communiqué «Il va en free, il a rien compris », écrit et diffusé par le collectif Mental Résistance et relayé régulièrement sur les réseaux spécialisés des teuffeurs et autres amateurs de free parties précise :

Le plus gros mensonge de nos soirées [est] de croire que les choses sont données, sans que ça coûte à qui que ce soit. En «free », c'est l'échange la valeur forte, c'est sur cette notion qu'est basée notre chère autogestion. Partager, troquer, de l'entraide, de la solidarité... être tous sur le même bateau, même si ce

\footnotetext{
${ }^{10}$ Entretien avec T., Bordeaux, 23 juillet 2018.

11 Entretien avec D., Tourettes, 29 décembre 2018.

12 Entretien avec M., Paris, 10 mars 2019.

$13 \mathrm{lbid}$.
} 
n'est que le temps d'une soirée. [...] Venir en « free party », c'est défendre une culture amateure sans profit, sans subvention mais qui a besoin quand même de s'autofinancer, pour pouvoir continuer à s'exprimer. On compte sur toi pour la donation ${ }^{14}$, si tu veux que cet esprit de « robin des bois culturel » perdure, et que d'autres « fêtes libres » puissent encore être proposées ${ }^{15}$.

Ce positionnement de la liberté de donner et d'aider chacun à sa mesure est présent depuis l'origine du mouvement, comme le rappelle un ex-deejay français actif entre 1995 et 2010 : «Chacun donne un peu, et le mouvement avance comme cela. L’idée c'est que tout le monde participe avec ses moyens ${ }^{16}$.»

La liberté d'aller et venir sans avoir à payer n'est pas la seule liberté alternative au néolibéralisme urbain festif. Le refus de tous les types de filtrage ou de sélection à l'entrée des soirées est au cœur de la notion même de free party, comme le confirment $78,9 \%$ des teuffeurs sondés pour qui la free party accepte tous les types de publics.

\begin{tabular}{|l|c|c|}
\hline & OUI & NON \\
\hline $\begin{array}{l}\text { Pensez-vous que les free } \\
\text { parties acceptent tous les } \\
\text { types de public? }\end{array}$ & $78,9 \%$ & $21,1 \%$ \\
\hline
\end{tabular}

(Échantillon de 1663 usagers de free party)

Ce refus de ségrégation à l'entrée des soirées est constitutif du mouvement free party. Il concerne autant la tenue vestimentaire que les moyens financiers et bien sûr la couleur de peau. T. se rappelle :

La culture free party réunissait tout le monde, elle a unifié toutes les communautés sur le dancefloor. Au tout début du mouvement, les gens venaient avec ce qu'ils étaient. Tu pouvais retrouver des meufs qui arrivaient en talons et sapées à nos soirées, des bourgeois qui venaient se faire peur à côtoyer des mauvais garçons, des jeunes issus des milieux ouvriers, des Antillais attirés par le breakbeat et la jungle. II y avait tout le monde dans nos soirées... ${ }^{17}$

Le constat est le même chez A. : «Il y avait plein de gens différents, j’ai même vu arriver des gars et des meufs en costard et en tailleur. Ils sortaient de boîte et cherchaient un after. C'était marrant parce que nous n'étions pas acceptés comme nous étions dans les lieux d'où ils venaient de faire la fête, mais nous les acceptions sans problème dans nos free... ${ }^{18}$ » B. renchérit : "Grâce à la musique, on permettait aux gens de se mélanger. On ouvrait les esprits ${ }^{19}$. " La Fuck Boréalis, teknival organisé dans le Gard en 1999 en

\footnotetext{
${ }^{14}$ Chaque participant donne le montant qu'il souhaite en arrivant en free party, même si cette donation reste au bon vouloir de chaque teuffeur.

${ }^{15}$ Communiqué du collectif Mental Résistance diffusé sur sa page Facebook le 11 avril 2019 et repris par de nombreux activistes et sound systems sur leurs réseaux sociaux. En ligne, consulté le 14 février 2020. URL : https://www.facebook.com/euphoriksound6tem/posts/2446950998759645.

${ }^{16}$ Entretien avec F., Marseille, 5 janvier 2019.

17 Entretien avec T., Bordeaux, 23 juillet 2018.

${ }^{18}$ Entretien avec A., Paris, 7 mars 2019.

${ }^{19}$ Entretien téléphonique avec B., 5 décembre 2018.
} 
marge de la Boréalis, archétype de la soirée électronique payante, est un bon exemple de ces mélanges. La free party voit débarquer une grande partie du public de la Boréalis, annulée en raison des conditions climatiques. Drag queens, fêtards habillés en fluo, jeunes femmes habillées en Jean-Paul Gaultier se mélangent sans aucun problème avec le public habituel des free parties.

La pratique du troc est un autre usage très développé en free party. Ainsi, 63,5 \% des teuffeurs sondés dans notre enquête y ont recours, principalement pour obtenir de l'alcool, de la nourriture et du cannabis. Les manières de pratiquer le troc sont multiples et variées, mais elles peuvent donner lieu à la mise en place de stratégies poussées. « À chaque teknival, on partait à Andorre, remplir la voiture de cartouches de cigarettes qui étaient notre principal outil de troc en teuf [...] avec ça, on était trop bien, on pouvait échanger de tout, de la bouffe, des mixtapes et le reste... », confie M. qui avait vingt ans en 2000, l'apogée de sa pratique des free parties ${ }^{20}$. La pratique du troc amène également certains participants à se lancer des défis pendant les trois jours de free party : "C'était l'idée de reprendre la légende urbaine du mec qui part avec un trombone et qui revient avec une voiture à force d'échanges. Sans aller jusque-là, c'était un délire de voir jusqu'où pouvait nous mener cette pratique qui remonte en fait au tout début de l'humanité ${ }^{21}$ », raconte $\mathrm{F}$. On retrouve la référence à une forme particulière de tribalité avec l'échange de services désintéressés (chose courante en free party) qui rappellent les notions de don de Marcel Mauss (2007 [1925]), que l'on peut envisager comme participant à la production de liens sociaux. « Le troc est une pratique qu'on a empruntée aux Amérindiens et aux pirates, mais c'était avant tout une nécessité. On ne voulait pas vivre sous le diktat de l'argent, alors on a développé au maximum l'échange, les solidarités, le troc et par la force des choses le DIY. On voulait tout faire de A à Z ${ }^{22}$ », explique T.

\begin{tabular}{|l|c|c|}
\hline & OUI & NON \\
\hline $\begin{array}{l}\text { Lors de ces free parties, y } \\
\text { pratiquez-vous/pratiquiez- } \\
\text { vous le troc? }\end{array}$ & $63,5 \%$ & $36,5 \%$ \\
\hline Pour quels types de produits : & & \\
\hline Alcool & $78,0 \%$ \\
\hline Nourriture & $75,0 \%$ \\
\hline Cannabis & $70,8 \%$ \\
\hline Drogues dures & $43,4 \%$ \\
\hline Disque/Mixtape & $18.5 \%$ \\
\hline Essence & $17,7 \%$ \\
\hline
\end{tabular}

(Échantillon de 1663 usagers de free parties)

\footnotetext{
${ }^{20}$ Entretien avec M., Paris, 10 mars 2019.

21 Entretien avec F., Marseille, 5 janvier 2019.

22 Entretien avec T., Bordeaux, 23 juillet 2018.
} 
Le DIY va en effet s'imposer comme une autre pratique emblématique des free parties, symbole de l'autogestion adoptée lors de l'organisation de fêtes libres. Rejetant les mécanismes de marchandisation et de l'ultra-consommation, basé sur la récupération et la débrouillardise, le DIY devient une pratique permettant de rester à l'extérieur de la société de consommation au maximum : " Ma fille est née en 1994, on avait rien et le DIY était une nécessité. On vivait dans la rue et on voulait montrer qu'on était capables de vivre différemment et d'assumer nos choix, comme celui d'avoir un enfant ${ }^{23}$ ", raconte $\mathrm{B}$. « Notre finalité n'était pas d'être contre le système, mais de rester en dehors du système. Dans cette logique, tu es obligé de tout créer par toi-même et donc le DIY tombe sous le sens. Tu ne peux pas faire autrement ${ }^{24}$ ", résume T. Les manières de s'habiller, d'habiter, et même de décorer les free parties sont basées sur la récupération. "On jouait la musique qu'on produisait dans les soirées qu'on organisait nous-mêmes, comme on le voulait, avec qui on le voulait», raconte M. L'argument du «à nous, par nous, pour nous » se retrouve ainsi dans les propos de tous les activistes du premier cercle des free parties : organisateurs ou membres de sound systems.

On retrouve d'ailleurs la référence à l'autogestion dans la structuration horizontale des sound systems à l'origine de l'organisation et/ou de l'animation des teknivals : pas de hiérarchie ou d'organisation pyramidale, partage des richesses créées, prise de décision collective. Dans la plupart des tribes, mécaniciens, cuisiniers, deejays, décorateurs sont tous membres du sound system à égal niveau d'importance. Il n'y a pas de chef déclaré, ni de hiérarchie à suivre. Chacun joue un rôle pour que le collectif fonctionne, musiciens comme techniciens, en fonction de leurs possibilités et de leurs moyens. Chacun participe aux prises de décisions de la tribe.

\begin{tabular}{|l|c|c|c|c|}
\hline & \multicolumn{2}{|c|}{ OUI } & \multicolumn{2}{c|}{ NON } \\
\hline $\begin{array}{l}\text { Y faites-vous/y } \\
\text { avez-vous fait } \\
\text { l'expérience de } \\
\text { l'autogestion? }\end{array}$ & \multicolumn{2}{|c|}{$94,1 \%$} & \multicolumn{2}{c|}{$\begin{array}{c}\text { Très } \\
\text { satisfaisante }\end{array}$} \\
\hline Pensez-vous que l'autogestion en matière de (...) est : & $\begin{array}{c}\text { Peu } \\
\text { satisfaisante }\end{array}$ & Satisfaisante \\
\hline $\begin{array}{l}\text { Qualité } \\
\text { musicale }\end{array}$ & Insuffisante & $53,4 \%$ & $30,1 \%$ \\
\hline $\begin{array}{l}\text { Approvisionne- } \\
\text { ment en drogue }\end{array}$ & $2,2 \%$ & $9,9 \%$ & $58,2 \%$ & $12,2 \%$ \\
\hline Sécurité & $3,1 \%$ & $19,4 \%$ & $65,3 \%$ & \\
\hline
\end{tabular}

${ }^{23}$ Entretien téléphonique avec B., 5 décembre 2018.
${ }^{24}$ Entretien avec T., Bordeaux, 23 juillet 2018. 


\begin{tabular}{|l|c|c|c|c|}
\hline $\begin{array}{l}\text { Nourriture } \\
\text { et eau }\end{array}$ & $2,8 \%$ & $19,1 \%$ & $59,3 \%$ & $18,8 \%$ \\
\hline Propreté & $13,4 \%$ & $34,7 \%$ & $38,7 \%$ & $13,2 \%$ \\
\hline
\end{tabular}

(Échantillon de 1663 usagers de free parties)

\section{Appropriation de l'espace, droit à la fête libre et TAZ : vers une géographie de la free party}

Les pratiques du troc, de l'autogestion, du do it yourself associées à l'organisation de fêtes libres s'incarnent dans des lieux où les initiateurs et les usagers de free parties s'approprient des espaces publics et privés pour créer des territoires alternatifs éphémères où s'exprime un droit à la fête libre.

Dans le cas de la free party, le mécanisme de réappropriation de l'espace peut ainsi s'entendre comme une contre-« production de l'espace » au sens de Henri Lefebvre (1974) et une réappropriation spatiale symbolique au sens de Pierre Bourdieu (1993). L'organisation d'une fête libre urbaine en dehors de tout encadrement normatif capitaliste et étatique apparaît en effet comme une contre-proposition territoriale idéelle et éphémère où l'affirmation et la revendication contre le néolibéralisme urbain festif sont manifestes. "On avait l'impression d'être au seul endroit où le monde dans lequel on croyait pouvait se réaliser. Et ce monde-là était l'opposé absolu de la société de consommation », explique C. ${ }^{25}$, vétéran du milieu free party. M. va plus loin, questionnant la propre réalité de ces lieux face à ses sensations : « Souvent en teuf, je me mettais un peu à l'écart et je me demandais si c'était vraiment ça la vie. J'étais trop bien dans ma tête et dans mon corps, mais partout autour de moi, c'était Mad $\mathrm{Max}^{26}$.»

Cette contre-production et réappropriation symbolique ne s'obtiennent pourtant pas selon le principe classique de luttes urbaines (Castells, 1977), où les mobilisations et les manifestations sont plutôt réalisées dans les centres-villes, au cœur des métropoles, puisque les acteurs et les usagers des free parties choisissent non pas des lieux de pouvoirs, mais des interstices urbains pour organiser leurs fêtes libres. «On allait squatter les hangars des magasins Monsieur Meuble qui venaient de faire faillite. On piquait les lettres des enseignes pour récrire des mots à nous, pour revendiquer notre refus du capitalisme $^{27}$ », raconte B. Ces espaces étaient le symbole des territoires laissés sur le bas-côté de la croissance néolibérale et de la mondialisation. Ils n’en acquéraient pas moins une charge symbolique forte à l'initiative des participants à la free party qui les marquaient de différentes manières, initiant ainsi un processus de territorialisation.

Le premier de ces marquages est l'organisation socioterritoriale des lieux par le positionnement des véhicules et des sound systems des organisateurs de la free party. L'appropriation de l'espace pouvait débuter une semaine avant la date de l'événement

\footnotetext{
${ }^{25}$ Entretien téléphonique avec C., 14 octobre 2018.

${ }^{26}$ Entretien avec M., Paris, 10 mars 2019.

27 Entretien téléphonique avec B., 5 décembre 2018.
} 
par le nettoyage des lieux, ce qui permettait de poser les bases de l'organisation des hangars ou des bâtiments squattés.

La disposition des murs de sons (empilement des enceintes du sound system) permettait de délimiter une première frontière visible de la TAZ. Elle en produisait une seconde, invisible mais terriblement efficace quand il s'agit de trouver le lieu de la fête en pleine nuit : la puissance sonore. « Le sentiment d'entrer dans un monde parallèle débutait dès qu'on entendait le son de la teuf. C'était le premier sas de décompression avant d'atteindre la free ${ }^{28}$ », explique F., activiste de la période 1996-2003. Une troisième, plus mobile, concernait le premier convoi de teuffeurs partant du lieu de rendez-vous diffusé par infoline (numéro de téléphone permettant de tomber sur un répondeur indiquant le chemin à suivre pour rejoindre la fête). Les organisateurs attendaient d'être assez nombreux pour ne pas pouvoir être ennuyés par les forces de police au cas où elles auraient souhaité empêcher la fête, interdire l'entrée du lieu choisi ou réquisitionner les sound systems. Un dernier marquage, enfin, permettait d'identifier la TAZ : l'enchaînement des voitures garées dans un parking sauvage souvent sans limites.

L'affirmation identitaire de la free party et de la TAZ se retrouve également au travers de différents drapeaux, bannières et décors particuliers (bâches militaires, matériel de camouflage, drapeaux de pirates, smileys). Elle constitue une autre manière d'identifier et de s'approprier les lieux. On la retrouve dans le look vestimentaire adopté par une partie des teuffeurs sur le modèle de celui des membres des tribes : crâne rasé, treillis, tee-shirts et pantalons noirs. «À la base, tout était pensé, on portait des vêtements sombres parce qu'ils marquaient moins la saleté, on se rasait la tête pour éviter les poux, la vie sur la route c'était avant tout être pragmatique... ${ }^{29}$ ", confie T. L'affirmation identitaire se veut ainsi concomitante d'une manière de se comporter et d'agir décrite dans la partie précédente qui aboutit à un changement de règles territorialisées : «On est en anarchie, on est dans un lieu à nous, pour nous, par nous ${ }^{30}$ », résume Y.

De ce point de vue, la free party peut s'appréhender comme un véritable droit à la fête libre, déclinaison du droit à la ville (Costes, 2010 ; 2014 ; Lefebvre, 1972). Il se positionne comme l'émanation de populations marginalisées en raison de leurs pratiques festives affirmant leur existence dans un acte de réquisition de territoires privés afin d'imposer et de pratiquer un certain nombre de libertés individuelles, de lutter contre la marchandisation de la ville et contre le néolibéralisme urbain.

Une approche géographique complète nous pousse néanmoins à aller au-delà de cette référence au droit à la ville, pour mobiliser la notion de TAZ développée par Hakim Bey (1991) afin d'analyser les mécanismes de territorialisation à l'œuvre lors de free parties. Nous proposons ainsi d'envisager la free party comme une TAZ hybride.

L'ensemble des pratiques festives alternatives que nous venons d'analyser peut ainsi s'entendre dans sa globalité comme un processus collectif de réappropriation ponctuelle de l'espace amenant une émancipation également ponctuelle du système

\footnotetext{
${ }^{28}$ Entretien avec F., Marseille, 5 janvier 2019.

${ }^{29}$ Entretien avec T., Bordeaux, 23 juillet 2018.

${ }^{30}$ Entretien avec Y., Nice, 18 octobre 2018.
} 
néolibéral pouvant se rapprocher de la notion de TAZ. Cette notion permet en effet d'aller au-delà des notions de droit à la ville, d'appropriation symbolique ou idéelle de l'espace en se définissant comme une « microsociété vivant délibérément hors-la-loi et bien déterminée à le rester, ne fût-ce que pour une vie brève, mais joyeuse » (Bey, 1991 : 5). « La TAZ, c'est chez nous, avec nos codes, nos potes, nos sonos, nos teufs. On fait tout comme on veut, le temps qu'on veut et on demande rien à personne ${ }^{31}$ ", résume $\mathrm{X}$., jeune activiste qui a fait sa première free en 2014 et qui depuis n'a plus lâché le mouvement.

Bey associe donc la posture antisystème à la proposition hédoniste d'une réalisation conjoncturelle mais intense des aspirations à la liberté. C'est exactement la posture d'une majorité des teuffeurs que nous avons interrogés lors de notre enquête, puisque fête libre et lieu festif sont les deux définitions largement retenues pour qualifier une free party. Il faut néanmoins préciser que la notion de TAZ, elle, a été peu utilisée par nos interlocuteurs lors des entretiens semi-directifs, et quand c'était le cas, ce sont les teuffeurs les plus jeunes qui l'employaient. Cette différence d'utilisation s'explique notamment par le fait que l'ouvrage de Bey a été écrit alors que le mouvement free party en était à ses balbutiements, l'auteur ne cite jamais ce mouvement dans son ouvrage sur les TAZ, et en raison de la méfiance des activistes de la première heure vis-à-vis de tout encadrement normatif (même en matière de vocabulaire).

\begin{tabular}{|l|c|c|c|c|c|c|}
\hline & $\begin{array}{c}\text { Une fête } \\
\text { libre }\end{array}$ & $\begin{array}{c}\text { Un lieu } \\
\text { festif }\end{array}$ & $\begin{array}{c}\text { Un } \\
\text { rassem- } \\
\text { blement } \\
\text { contesta- } \\
\text { taire }\end{array}$ & $\begin{array}{c}\text { Une fête } \\
\text { illégale }\end{array}$ & $\begin{array}{c}\text { Un } \\
\text { supermar- } \\
\text { ché de la } \\
\text { drogue }\end{array}$ & $\begin{array}{c}\text { Un lieu } \\
\text { dangereux }\end{array}$ \\
\hline $\begin{array}{l}\text { Comment } \\
\text { définiriez- } \\
\text { vous une } \\
\text { free party? } \\
\text { (choix } \\
\text { multiples) }\end{array}$ & $92,1 \%$ & $69,7 \%$ & $29,1 \%$ & $21,4 \%$ & $10,1 \%$ & $2,0 \%$ \\
\hline
\end{tabular}

(Échantillon de 1663 usagers de free parties)

Dans cette perspective, la TAZ dépasse la simple remise en cause du néolibéralisme urbain festif pour être force de proposition territoriale. La TAZ va au-delà de la révolution et de ses impacts, estimant qu'un « choc frontal avec l'État terminal, l'État de l'information méga-entrepreneurial, l'empire du Spectacle et de la Simulation, ne produirait absolument rien, si ce n'est quelques martyres futiles »(Bey, 1991: 8). Et si la TAZ s'envisage au-delà de la révolution, c'est que, pour Bey, le soulèvement, le mouvement de révolte lui-même est l'incarnation propre de la liberté, sous-entendant que la liberté n'est pas un état humain pérenne, mais plutôt un moment territorialisé particulier à

${ }^{31}$ Entretien téléphonique avec X., 4 décembre 2018. 
saisir. « Il fallait vivre vite et intensément, puis bouger pour recréer une nouvelle île de liberté. Nous étions des nomades de la fête ${ }^{32} »$, illustre Y.

C'est dans ce contexte qu'une analyse du mouvement free party à travers la notion de TAZ renvoie ses membres à la notion de psychotopologie révélatrice des aspirations des teuffeurs. Bey précise le terme qu'il invente pour qualifier la recherche d'espaces-temps libres :

Nous recherchons des « espaces » (géographiques, socio-culturels, imaginaires) capables de s'épanouir en zones autonomes - et des espaces-temps durant lesquels ces zones sont relativement ouvertes, soit du fait de la négligence de l'État, soit qu'elles aient échappé aux arpenteurs ou pour quelque autre raison encore. La psychotopologie est l'art du sourcier des TAZ potentielles. (Bey, 1991 : 11)

Si l'on suit ce raisonnement, les usagers et les initiateurs des free parties apparaissent alors comme des psychotopologues qui s'ignorent. La notion de TAZ permet ainsi d'appréhender la free party non pas seulement comme une émanation d'une lutte hypothétique contre le néolibéralisme urbain festif mais également comme la finalité territoriale des aspirants fêtards à la pratique d'un autre type de fête libre et solidaire. La free party devient selon $\mathrm{T}$. un « microsystème parallèle où toutes les règles du système dominant étaient bannies et remplacées par nos propres règles et aspirations ${ }^{33}$ ॥. Et même si l'affirmation de cette liberté territoriale radicale et absolue doit toutefois être nuancée par la présence d'un certain nombre d'éléments incontrôlables (par exemple, les dealers extérieurs à la philosophie de la free party, l'envisageant comme un supermarché de la drogue leur permettant de faire des bénéfices) ou de comportements toxico-addictifs (la prise de certains psychotropes peut être plus contraignante à terme que libératrice), elle demeure un élément constitutif de la culture sound system.

C'est probablement pour ces raisons que le rapport de force avec les pouvoirs publics n'a cessé de se tendre, notamment en France. Depuis l'amendement Mariani voté en $2001^{34}$ et soumettant les free parties à une déclaration préalable en préfecture, jusqu'à la récente proposition de loi de la sénatrice Pascale Bories $(2018)^{35}$ visant à durcir encore plus l'arsenal judiciaire répressif, les gouvernements successifs ont principalement joué la carte de la sanction contre le mouvement free party. Celle-ci s'est incarnée par de nombreuses arrestations, des gardes à vue, des saisies de matériels sonores, des condamnations à de lourdes amendes, parfois même à de la prison ferme. Dans ce contexte d'opposition frontale avec les autorités, le rapport des teuffeurs à la police est loin d'être pacifié. Lors de notre enquête, seulement 3,5\% des teuffeurs interrogés estimaient que la présence de la police les rassurait, et 21,7 \% qu'elle permettait de sécuriser la zone. Ils sont en revanche $47 \%$ à estimer que la police ne sert à rien dans une free party et 53,3 \% à penser qu'elle provoque. Les policiers sont également le deuxième pu-

\footnotetext{
32 Entretien avec Y., Nice, 18 octobre 2018.

${ }^{33}$ Entretien avec T., Bordeaux, 23 juillet 2018.

${ }^{34}$ Amendement à la loi sur la sécurité quotidienne votée le 15 novembre 2001.

35 Proposition de loi enregistrée le 10 septembre 2018 tendant à renforcer l'encadrement des rave parties et les sanctions à l'encontre de leurs organisateurs. En ligne, consulté le 15 février 2020. URL : https://www.senat.fr/leg/ppl17-711.html.
} 
blic jugé indésirable en free party, à $41,3 \%$, juste derrière les... « voyous » (définis comme les personnes qui commettent des activités délictueuses), à 54,3\%. Le positionnement des teuffeurs vis-à-vis de la police est donc clairement affirmé : ils sont les empêcheurs de faire la fête de manière libre, d'écouter de la musique comme ils le souhaitent et de diffuser leur message partout où ils le désirent.

Il est d'ailleurs important de noter à ce sujet qu'il n'y a pas eu de théorisation politique sur l'organisation des free parties, ni de texte écrit par ses fondateurs pour donner une vision a priori ou proposer une réinterprétation a posteriori du positionnement politique des teuffeurs :

Comme c'était notre vie, la façon dont on vivait tous les jours, on avait aucune justification à donner. On écoutait de la musique $24 \mathrm{~h} / 24 \mathrm{~h}$. On voulait pas s'arrêter de danser. C'est de là qu'est venue l'idée que le message était dans la musique et qu'il se diffusait à partir d'un petit nombre de gens comme une spirale pouvait se diffuser d'un centre vers le reste de l'univers ${ }^{36}$.

\section{Conclusion}

Depuis quarante ans, la géographie des nuits urbaines festives a évolué sous l'effet du néolibéralisme. La nuit se vend, et elle fait vendre. Les interdictions, la sélection socioéconomique, le filtrage, la marchandisation des pratiques festives se diffusent dans toutes les villes, et ce d'autant plus que la fête est envisagée comme un nouveau vecteur de régénération urbaine.

Dans ce contexte, la free party s'est positionnée comme une alternative au néolibéralisme festif en se fondant sur des principes opposés de liberté d'accès, de non-marchandisation, d'autogestion, de DIY et de troc. Elle est apparue de fait comme une déclinaison festive du droit à la ville. Mais au-delà d'une contestation libertaire éphémère, elle remet en question l'expression classique des luttes urbaines pour instituer de manière ponctuelle des TAZ dédiées à la fête. La révolution ou le changement social ne sont pas les objectifs affirmés de ce processus de territorialisation original, c'est le moment de lutte lui-même et son euphorie qui deviennent la finalité de l'action contestataire, laissant présager une mutation en cours des formes d'expression subversives opposées à la ville et à la société néolibérales.

Par ailleurs, près de trente ans après l'apparition des free parties, l'organisation encore régulière de fêtes électroniques libres un peu partout en France s'inscrit désormais dans un contexte de multiplication des TAZ, en particulier sous la forme de zones à défendre $(\mathrm{ZAD})$. Ces zones à défendre, dont la plus emblématique a été Notre-Damedes-Landes, possèdent de nombreuses similitudes en ce qui concerne leur organisation, leurs usagers et leur territorialisation. Elles participent clairement à l'affirmation de nouvelles formes de luttes sociales, s'incarnant dans des territoires éphémères où les règles dominantes sont remises en cause et où des espaces de sociabilisations nouvelles émergent. De ce point de vue là, l'occupation illégale récente des ronds-points lors du

${ }^{36}$ Entretien avec T., Bordeaux, 23 juillet 2018. 
mouvement des Gilets jaunes peut également s'envisager comme une nouvelle forme de TAZ et constitue un véritable challenge pour l'étude de la géographie des mouvements sociaux et des luttes urbaines.

\section{Bibliographie}

Ballas D. (2013), « What Makes a Happy City », Cities, vol. 1, n 9, p. 39-50.

Baudrillard J. (1997), Écran total, Paris, Galilée.

Bell D. (2008), « Destination Drinking: Toward a Research Agenda on Alcotourism », Drugs: Education, Prevention and Policy, n 15, p. 291-304.

Bey H. (1991), Taz (zone d'autonomie temporaire), Paris, Éditions de l'éclat.

Bonnemaison S. (1990), « City Policies and Cyclical Events », Design Quaterly, n 147, p. 24 32.

Bourdieu P. (1993), « Effets de lieu », in P. Bourdieu (dir.), La misère du monde, Paris, Seuil, p. 159-165.

Bouyxou J.-P., Delannoy P. (1995), L'aventure hippie, Paris, Le Lézard.

Brenner N., Theodore N. (2002), "Cities and the Geographies of "Actually Existing Neoliberalism" ", Antipode, vol. 34, n 3, p. 349-379.

Burgel G. (1993), La ville aujourd'hui, Paris, Hachette Pluriel.

Burgel G., Grondeau A. (2015), Géographie urbaine, Paris, Hachette Éducation.

CAmprubi R., Prats L. (2013), «The Perceived Image of Barcelona by Short Break Tourists », 1st Annual International Interdisciplinary Conference, AIIC 2013, 24-26 April, Azores, Portugal, p. 941-949.

Capellà i Miternique H. (2017), « The Ibiza's Nightlife as a Bend from Marginalization to Tourism Centrality », in S. Pelc, M. Koderman (eds), Nature, Tourism and Ethnicity as Drivers of (De)Marginalizatin, London, Springer, p. 109-118.

Carroué L. (2002), Géographie de la mondialisation, Paris, Colin.

Castells M. (1977), « Les conditions d'émergence des mouvements sociaux urbains. À partir d'une enquête exploratoire sur les luttes urbaines dans la région parisienne, 1968-1973 », International Journal of Urban and Regional Research, vol. 1, n 1-3, p. 4775.

Chatterton P., Hollands R. (2003 ), Urban Nightscapes: Youth Cultures, Pleasure Spaces and Corporate Power, London, Routledge.

Collette T. (2002), « Une identité collective : les free parties », Revue du MAUSS, vol. 19, $\mathrm{n}^{\circ} 1, \mathrm{p} .349-356$.

Costes L. (2010), « Le Droit à la ville de Henri Lefebvre : quel héritage politique et scientifique ?», Espaces et sociétés, n 140-141, p.177-191. 
Costes L. (2014), « Néolibéralisation et évolution du "Droit à la ville” », Justice spatialeSpatial justice, $\mathrm{n}^{\circ}$ 6. En ligne, consulté le 22 décembre 2019. URL : https://www.jssj. org/article/neoliberalisation-et-evolution-du-droit-a-la-ville/.

Crozat D., Fournier L. (2005), « De la fête aux loisirs : événement, marchandisation et invention des lieux », Annales de géographie, vol. 643, n³, p. 307-328.

Debord G. (1992 [1967]), La société du spectacle, Paris, Gallimard.

Epstein R. (2001), " Les raves ou la mise à l'épreuve underground de la centralité parisienne », Mouvements, vol. 13, n 1, p. 73-80.

Di Meo G. (2005), « Le renouvellement des fêtes et des festivals, ses implications géographiques », Annales de géographie, vol. 643, n 3, p. 227-243.

FJeR E.G., Tutenges S. (2017), « Departies: Conceptualizing Extended Youth Parties », Journal of Youth Studies, vol. 20, n² 2, p. 200-215.

GicQuel C. (2007), «Free party : une aire de Je(u) dans l'air du temps », Espace populations sociétés, $\mathrm{n}^{\circ} 2-3$, p. 345-356.

Giordano E, Nofre Mateu J., Crozat D. (2018), « La touristification de la vie nocturne : une nouvelle frontière pour la recherche sur la nuit urbaine », Cybergeo : revue européenne de géographie, $\mathrm{n}^{\circ}$ 866. En ligne, consulté le 22 décembre 2019. URL : http://journals. openedition.org/cybergeo/29473.

Grataloup C. (2015), Géohistoire de la mondialisation, Paris, Armand Colin.

Gravari-Barbas M. (2000), La ville festive. Espaces, expressions, acteurs, Habilitation à diriger des recherches, Angers, Université d'Angers.

GraVARI-BARBAS M. (2009), " La "ville festive” ou construire la ville contemporaine par l'événement », Bulletin de l'Association de géographes français, vol. 86, p. 279-290.

Gwiazdzinski L. (2007), " L’archipel des mobilités nocturnes », Données urbaines, n 5 , p. 87-96.

Gwiazdzinski L. (2014), "Pleading for the right to the city's night », Night Manifesto Seeking Citizenship 24h, Sao Paulo, Invisiveis Produçoes, p. 204-220.

GwiazDZinski L. (2015), « The Urban Night: A Space Time for Innovation and Sustainable Development », Articulo. Journal of Urban Research, vol. 11, p. 1-15.

HaRvey D. (1989), « From Managerialism to Entrepreneurialism: the Transformation in Urban Governance in Late Capitalism », Geografiska Annaler, vol. 71, n 1, p. 3-17.

HaRvey D. (2014), Géographie de la domination, Paris, Les Prairies Ordinaires.

Kosmicki G. (2008), « Transe, musique, liberté, autogestion », Cahiers d'ethnomusicologie, $\mathrm{n}^{\circ}$ 21. En ligne, consulté le 23 décembre 2019. URL : http://ethnomusicologie.revues. org $/ 1185$.

Lacour C., Puissant S. (2000), La métropolisation - Croissance, diversité et fractures, Paris, Anthropos-Economica («Villes »). 
Lefebvre H. (1972), Le droit à la ville, suivi de Espace et politique, Paris, Anthropos.

Lefebvre H. (1974), La production de l'espace, Paris, Economica.

MafFesoli M. (1993), La contemplation du monde : figures du style communautaire, Paris, Grasset et Fasquelle.

Mauss M. (2007 [1925]), Essai sur le don. Forme et raison de l'échange dans les sociétés archaïques, Paris, Presses universitaires de France ("Quadrige Grands textes »).

Oloukoï C., GuinaRd P. (2016), « La nuit à Maboneng (Johannesburg, Afrique du Sud) : un front urbain entre sécurisation, marchandisation et contestation », L'Espace Politique, $\mathrm{n}^{\circ}$ 30. En ligne, consulté le 23 décembre 2019. URL : http://journals. openedition.org/espacepolitique/3977.

PECK J. (2014), « Entrepreneurial Urbanism: Between Uncommon Sense and Dull Compulsion », Geografiska Annaler, vol. 96, n² 4, p. 396-401.

Petiau A. (2006), « Marginalité et musiques électroniques », Agora débats/jeunesses, $\mathrm{n}^{\circ} 42$, p.128-139.

Petiau A. (2011), « L'“âme tigrée” des musiques électroniques. Les imaginaires des jeunes et les courants musicaux », Sociétés, vol. 112, nº 2, p. 115-122.

POURTAU L. (2005), « Les interactions entre raves et législations censées les contrôler », Déviance et Société, vol. 29, n², p. 127-139.

QueudRus S. (2001), « La free-party, sociologie d'un vagabondage festif », Quaderni, $\mathrm{n}^{\circ} 44$, p. 129-142.

RENARD V. (2008), «La ville saisie par la finance », Le Débat, vol. 1, n¹48, p. 106-117.

StraW W. (2001), « Scenes and Sensibilities », Public, vol. 22/23, p. 245-257.

Tessier L. (2003), « Musiques et fêtes techno : l'exception franco-britannique des free parties », Revue française de sociologie, vol. 44, n 1, p. 63-91.

WARD K. (2003), « Entrepreneurial Urbanism, State Restructuring and Civilizing "New” East Manchester », Area, vol. 35, n² 2, p. 116-127. 